\title{
A BIMA ARRAY SURVEY OF MOLECULES IN COMETS LINEAR (C/2002 T7) AND NEAT (C/2001 Q4)
}

\author{
Anthony J. Remijan, ${ }^{1,2,3}$ D. N. Friedel, ${ }^{4}$ Imke de Pater, ${ }^{5}$ M. R. Hogerheijde, ${ }^{6}$ L. E. Snyder, ${ }^{4}$ M. F. A'Hearn, ${ }^{7}$ Geoffrey A. Blake, ${ }^{8}$ \\ H. R. Dickel, ${ }^{4,9}$ J. R. Forster, ${ }^{5}$ C. Kraybill, ${ }^{5}$ L. W. Looney, ${ }^{4}$ Patrick Palmer, ${ }^{10}$ and M. C. H. Wright ${ }^{5}$ \\ Received 2005 December 30; accepted 2006 January 26
}

\begin{abstract}
We present an interferometric search for large molecules, including methanol $\left(\mathrm{CH}_{3} \mathrm{OH}\right)$, methyl cyanide $\left(\mathrm{CH}{ }_{3} \mathrm{CN}\right)$, ethyl cyanide $\left(\mathrm{CH}_{3} \mathrm{CH}_{2} \mathrm{CN}\right)$, ethanol $\left(\mathrm{CH}_{3} \mathrm{CH}_{2} \mathrm{OH}\right)$, and methyl formate $\left(\mathrm{CH}_{3} \mathrm{OCHO}\right)$, in comets LINEAR $(\mathrm{C} / 2002$ T7) and NEAT (C/2001 Q4) with the Berkeley-Illinois-Maryland Association (BIMA) array. We also searched for transitions of the simpler molecules $\mathrm{CS}, \mathrm{SiO}, \mathrm{HNC}, \mathrm{HN}^{13} \mathrm{C}$, and ${ }^{13} \mathrm{CO}$. We detected transitions of $\mathrm{CH}_{3} \mathrm{OH}$ and $\mathrm{CS}$ around comet LINEAR and one transition of $\mathrm{CH}_{3} \mathrm{OH}$ around comet NEAT within a synthesized beam of $\sim 20^{\prime \prime}$. We calculated the total column density and production rate of each molecular species using the variable temperature and outflow velocity (VTOV) model recently described by Friedel et al. Considering the molecular production rate ratios with respect to water, comet T7 LINEAR is more similar to comet Hale-Bopp, while comet Q4 NEAT is more similar to comet Hyakutake. It is unclear, however, due to such a small sample size, whether there is a clear distinction between a Hale-Bopp and Hyakutake class of comet or whether comets have a continuous range of molecular production rate ratios.
\end{abstract}

Subject headings: comets: individual (LINEAR (C/2002 T7), NEAT (C/2001 Q4)) — molecular processes — radio lines: solar system — techniques: interferometric

\section{INTRODUCTION}

Comets are believed to contain the most pristine material remaining from the presolar nebula and are providing important insights into the formation mechanisms of complex molecular species. Comets are primarily located in two distinct regions of the solar system. The Oort Cloud, the source of long-period $(P>$ $200 \mathrm{yr}$ ) comets, is a spherically symmetric distribution of comets that encompasses the solar system out to a distance of nearly 100,000 AU. The Kuiper-Edgeworth Belt that lies in the ecliptic plane just beyond the orbit of Pluto out to several hundred AU is the source of short-period (20 yr $<P<200 \mathrm{yr}$ ) comets (Jewitt 2004). Recent models have suggested that the Oort Cloud comets may have had origins in the entire giant planet region between Jupiter and Neptune. These models suggest that comets were formed in a much wider range of nebular environments and probably experienced thermal and collisional processing before they were ejected into the Oort Cloud (Weissman 1999). This pro-

\footnotetext{
1 NASA Goddard Space Flight Center, Computational and Information Sciences and Technologies Office, Code 606, Greenbelt, MD 20771.

2 National Research Council Resident Research Associate.

3 Current address: National Radio Astronomy Observatory, 520 Edgemont Road, Charlottesville, VA 22901; aremijan@nrao.edu.

4 Department of Astronomy, 1002 West Green Street, University of Illinois, Urbana, IL 61801; friedel@astro.uiuc.edu, snyder@astro.uiuc.edu, lwl@astro .uiuc.edu.

5 Department of Astronomy, University of California, Berkeley, CA 94720; imke@floris.berkeley.edu, rforster@astro.berkeley.edu, ckraybill@astro.berkeley .edu, wright@astro.berkeley.edu.

${ }^{6}$ Leiden Observatory, P.O. Box 9513, 2300 RA, Leiden, Netherlands; michiel@strw.leidenuniv.nl.

7 Department of Astronomy, University of Maryland, College Park, MD 20742-2421; ma@astro.umd.edu.

${ }^{8}$ Division of Geological and Planetary Sciences, Division of Chemistry and Chemical Engineering, California Institute of Technology 150-21, Pasadena, CA 91125; gab@gps.caltech.edu.

9 Current address: Department of Physics and Astronomy, University of New Mexico, 800 Yale Boulevard NE, Albuquerque, NM 87131; h-dickel@phys .unm.edu.

${ }^{10}$ Department of Astronomy and Astrophysics, 5640 South Ellis Avenue, University of Chicago, Chicago, IL 60637; ppalmer@oskar.uchicago.edu.
}

cessing may have "homogenized" the cometary nuclei of Oort Cloud comets. Observations comparing long- and short-period comets, which are believed to have had different origins, will allow us to address the molecular diversity in comets (A'Hearn et al. 1995; Mumma et al. 2005).

Furthermore, a key goal in astrochemistry is to learn whether the molecular diversity seen in high- and low-mass hot molecular cores (HMCs) is similar to the chemistry of the primordial solar nebula. The molecular diversity in comets may provide the link between the formation mechanisms of complex molecular species seen in high-mass HMCs and low-mass young stellar objects and the formation of young solar systems. Therefore, only through comprehensive surveys of HMCs and comets will a consistent, coherent picture emerge regarding the basic chemistry involved in the evolution of HMCs and comets.

Most observations of the molecular composition of cometary gas and dust have taken place in the visible, ultraviolet (see e.g., Hutsemékers et al. [2005] and references therein), or infrared parts of the spectrum (see, e.g., Helbert et al. 2005; Dello Russo et al. 2001). A vast inventory of interstellar ices and volatiles was also discovered by satellites passing through the coma of comet Halley (see, e.g., Mitchell et al. 1992). However, there have been several successful detections of the rotational transitions of molecular species, including organic compounds, at millimeter wavelengths (see, e.g., Crovisier et al. 2004a, 2004b; Biver et al. 2002). Many of these species are important in prebiotic organic chemistry (e.g., $\mathrm{H}_{2} \mathrm{O}, \mathrm{HCN}, \mathrm{CH}_{3} \mathrm{OH}$, aldehydes, and nitriles) and are believed to be parent molecules (originating from the comet nucleus), rather than the products of photodissociation or gas-phase chemistry in the cometary coma. Two prominent examples of parent molecules of prebiotic importance are hydrogen cyanide ( $\mathrm{HCN}$; see, e.g., Friedel et al. 2005) and methanol $\left(\mathrm{CH}_{3} \mathrm{OH}\right.$; see, e.g., Ikeda et al. 2002).

In the spring of 2004, there was a rare opportunity to observe two dynamically new Oort Cloud comets passing into the inner solar system and within $\sim 0.3 \mathrm{AU}$ of the Earth: comet LINEAR (C/2002 T7, hereafter T7 LINEAR) and comet NEAT (C/2001 Q4, hereafter Q4 NEAT). This paper presents the results of an 
TABLE 1

Molecular Line Parameters

\begin{tabular}{|c|c|c|c|c|c|}
\hline $\begin{array}{l}\text { Species } \\
\text { (1) }\end{array}$ & $\begin{array}{c}\text { Quantum Numbers } \\
\text { (2) }\end{array}$ & $\begin{array}{l}\text { Frequency } \\
\text { (MHz) } \\
\text { (3) }\end{array}$ & $\begin{array}{c}E_{u} \\
(\mathrm{~K}) \\
(4)\end{array}$ & $\begin{array}{l}S \mu^{2} \\
\left(D^{2}\right) \\
(5)\end{array}$ & $\begin{array}{c}\alpha(1 \mathrm{AU})^{\mathrm{a}} \\
\left(\mathrm{s}^{-1}\right) \\
(6)\end{array}$ \\
\hline $\mathrm{CH}_{3} \mathrm{OH} \ldots \ldots \ldots \ldots \ldots \ldots$ & $3_{1,3}-4_{0,4} A^{+}$ & $107,013.770(13)$ & 28.3 & 3.1 & $1.3 \times 10^{-5}$ \\
\hline CS & $2-1$ & $97,980.985(15)$ & 7.7 & 7.1 & $6.7 \times 10^{-6}$ \\
\hline${ }^{13} \mathrm{CO}$ & $1-0$ & $110,201.353(1)$ & 5.3 & 0.01 & $1.2 \times 10^{-6 b}$ \\
\hline $\mathrm{CH}_{3} \mathrm{CN}$ & $6, \mathrm{~K}-5, \mathrm{~K}$ & $110,383.502(2)^{\mathrm{c}}$ & 18.5 & 91.9 & $2.9 \times 10^{-6}$ \\
\hline $\mathrm{CH}_{3} \mathrm{CH}_{2} \mathrm{CN}$ & $10_{1,10}-9_{1,9}$ & $86,819.846(0)$ & 24.1 & 146.7 & $\sim 10^{-5}$ \\
\hline $\mathrm{SiO}$ & $2-1$ & $86,846.960(50)$ & 6.3 & 19.2 & $6.7 \times 10^{-6}$ \\
\hline $\mathrm{HN}^{13} \mathrm{C}$ & $1-0$ & $87,090.850(50)$ & 4.2 & 7.3 & $1.5 \times 10^{-5 \mathrm{~d}}$ \\
\hline $\mathrm{CH}_{3} \mathrm{CH}_{2} \mathrm{OH}$ & $4_{1,4}-3_{0,3}$ & $90,117.576(2)$ & 9.4 & 5.4 & $1.8 \times 10^{-5}$ \\
\hline \multirow[t]{2}{*}{$\mathrm{CH}_{3} \mathrm{OCHO}$} & $8_{0,8}-7_{0,7} E$ & $90,227.595(13)$ & 20.1 & 21.0 & $4.7 \times 10^{-5}$ \\
\hline & $8_{0,8}-7_{0,7} A$ & $90,229.647(14)$ & 20.1 & 21.0 & $4.7 \times 10^{-5}$ \\
\hline 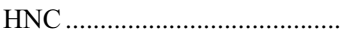 & $1-0$ & $90,663.572(4)$ & 4.4 & 9.3 & $1.5 \times 10^{-5}$ \\
\hline
\end{tabular}

Noтеs._All molecular line parameters taken from Pickett et al. (1998). Errors in the rest frequency are $2 \sigma$.

a Taken or estimated from Crovisier et al. (1994).

${ }^{b}$ Assumed to be the same as for CO.

${ }^{c}$ Only the frequency of the $K=0$ transition is given.

d Assumed to be the same as for HNC.

effort to further investigate the astrochemistry of comets by observing several large molecular species in comets T7 LINEAR and Q4 NEAT with the Berkeley-Illinois-Maryland Association (BIMA) array; ${ }^{11}$ these species include methanol $\left(\mathrm{CH}_{3} \mathrm{OH}\right)$, methyl cyanide $\left(\mathrm{CH}_{3} \mathrm{CN}\right)$, ethyl cyanide $\left(\mathrm{CH}_{3} \mathrm{CH}_{2} \mathrm{CN}\right)$, ethanol $\left(\mathrm{CH}_{3} \mathrm{CH}_{2} \mathrm{OH}\right)$, and methyl formate $\left(\mathrm{CH}_{3} \mathrm{OCHO}\right)$. In addition, we also searched for transitions of the simpler molecules $\mathrm{CS}$, $\mathrm{SiO}, \mathrm{HNC}, \mathrm{HN}^{13} \mathrm{C}$, and ${ }^{13} \mathrm{CO}$. We were successful in obtaining single-field images, cross-correlation spectra, and production rates for cometary methanol $\left(\mathrm{CH}_{3} \mathrm{OH}\right)$ in both comets and $\mathrm{CS}$ in comet T7 LINEAR. Upper limits were found for the remaining species in both comets.

\section{OBSERVATIONS AND RESULTS}

The observations were conducted using the BIMA array near Hat Creek, California, ${ }^{12}$ in D configuration (baselines from $\sim 6$ to $\sim 35 \mathrm{~m}$ ), cross-correlation mode toward comets T7 LINEAR and Q4 NEAT during their 2004 apparitions.

\footnotetext{
11 Operated by the University of California, Berkeley, the University of Illinois, and the University of Maryland with support from the National Science Foundation.
}

${ }^{12} 121^{\circ} 28^{\prime} 8^{\prime \prime} 0$ west, $40^{\circ} 49^{\prime} 4^{\prime \prime} 1$ north; altitude $1033 \mathrm{~m}$.
The observations of comet T7 LINEAR, using JPL reference orbit 69, were taken 2004 May 11-15 near $\alpha=01^{\mathrm{h}} 40^{\mathrm{m}}, \delta=$ $-09^{\circ} 30^{\prime}(\mathrm{J} 2000.0) .^{13}$ The comet was at a heliocentric distance of $0.73-0.77 \mathrm{AU}$ and a geocentric distance of $0.32-0.44 \mathrm{AU}\left(1^{\prime \prime}=\right.$ $319 \mathrm{~km}$ at $0.44 \mathrm{AU})$. W3 $(\mathrm{OH})$ was used as the flux density calibrator for these observations. The quasar $0108+015$ was used to calibrate the antenna based gains. The observations of comet Q4 NEAT, using JPL reference orbit 123, were taken 2004 May 20 24 near $\alpha=09^{\mathrm{h}} 07^{\mathrm{m}}, \delta=34^{\circ} 30^{\prime 14}(\mathrm{~J} 2000.0)$. The comet was at a heliocentric distance of $\sim 0.97 \mathrm{AU}$ and a geocentric distance of $0.55-0.61 \mathrm{AU}\left(1^{\prime \prime}=442 \mathrm{~km}\right.$ at $\left.0.61 \mathrm{AU}\right)$. Mars was used as the flux density calibrator, and $0927+390$ was used to calibrate the antenna based gains. The absolute amplitude calibration is accurate to within $\sim 20 \%$. The channel spacing of all observations was $0.391 \mathrm{MHz}$, except for CS, which had a spacing of $0.098 \mathrm{MHz}$. The data were reduced, combined, and imaged using the MIRIAD software package (Sault et al. 1995).

Table 1 lists the molecular line parameters of the searched species in comets T7 LINEAR and Q4 NEAT. Column (1) lists the

\footnotetext{
13 Both comets moved substantially across the sky during the observations;

thus, only approximate coordinates are given.
${ }_{14}$ See footnote 13 .
}

TABLE 2

Observational Parameters

\begin{tabular}{|c|c|c|c|c|c|c|}
\hline \multirow[b]{2}{*}{ SPECIES } & \multicolumn{3}{|c|}{ LINEAR } & \multicolumn{3}{|c|}{ NEAT } \\
\hline & $\begin{array}{c}r_{H} \\
(\mathrm{AU})\end{array}$ & $\begin{array}{c}\Delta \\
(\mathrm{AU})\end{array}$ & $\begin{array}{c}\theta_{a} \times \theta_{b} \\
\quad(\times)\end{array}$ & $\begin{array}{c}r_{H} \\
(\mathrm{AU})\end{array}$ & $\begin{array}{c}\Delta \\
(\mathrm{AU})\end{array}$ & $\begin{array}{c}\theta_{a} \times \theta_{b} \\
(\times)\end{array}$ \\
\hline $\mathrm{CH}_{3} \mathrm{OH}$ & 0.75 & 0.38 & $22.3 \times 16.9$ & 0.97 & 0.55 & $16.7 \times 14.3$ \\
\hline $\mathrm{CS}$ & 0.74 & 0.41 & $23.7 \times 18.9$ & 0.97 & 0.55 & $18.8 \times 15.4$ \\
\hline${ }^{13} \mathrm{CO}$ & 0.75 & 0.38 & $21.8 \times 16.1$ & 0.97 & 0.55 & $16.0 \times 14.1$ \\
\hline $\mathrm{CH}_{3} \mathrm{CN}$ & 0.75 & 0.38 & $21.6 \times 16.4$ & 0.97 & 0.55 & $16.2 \times 14.0$ \\
\hline $\mathrm{CH}_{3} \mathrm{CH}_{2} \mathrm{CN}$ & 0.77 & 0.32 & $28.5 \times 21.3$ & & & \\
\hline $\mathrm{SiO}$ & 0.77 & 0.32 & $28.5 \times 21.3$ & & & \\
\hline $\mathrm{HN}^{13} \mathrm{C}$ & 0.77 & 0.32 & $28.7 \times 20.0$ & & & \\
\hline $\mathrm{CH}_{3} \mathrm{CH}_{2} \mathrm{OH}$ & 0.77 & 0.32 & $28.2 \times 19.6$ & & & \\
\hline $\mathrm{CH}_{3} \mathrm{OCHO}$ & 0.77 & 0.32 & $27.7 \times 19.3$ & & & \\
\hline HNC & 0.77 & 0.32 & $27.7 \times 20.4$ & & & \\
\hline
\end{tabular}



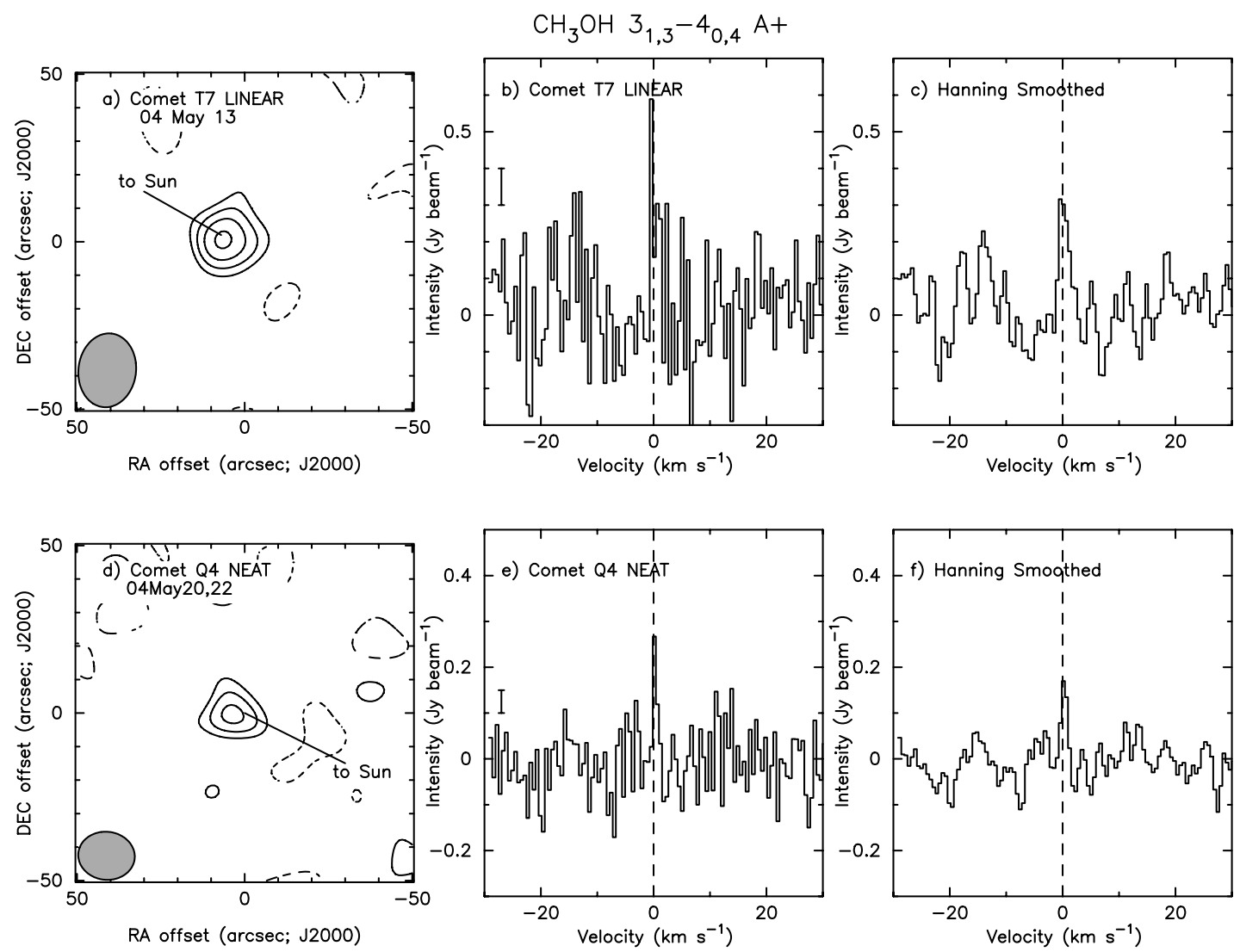

FIg. 1. - Comet LINEAR (C/2002 T7) and NEAT (C/2001 Q4) single-field $\mathrm{CH}_{3} \mathrm{OH}$ images and spectra. (a) Comet T7 emission contours from the $J=3_{1,3}-4_{04} A^{+}$ transition of $\mathrm{CH}_{3} \mathrm{OH}$ at $107.013 \mathrm{GHz}$. The contour levels are $-0.2,0.3,0.4,0.5$, and $0.6 \mathrm{Jy}$ beam ${ }^{-1}(1 \sigma$ spacing, starting at $3 \sigma)$. Image coordinates are arcsecond offsets relative to the predicted position of the nucleus. The synthesized beam is in the lower left, and the line segment shows the solar direction. $(b) \mathrm{CH}_{3} \mathrm{OH}$ cross-correlation spectra. Ordinate is flux density per beam, $I_{\nu}$, in Jy beam ${ }^{-1}, \sigma \sim 0.1 \mathrm{Jy} \mathrm{beam}^{-1}$. (c) $\mathrm{CH}_{3} \mathrm{OH}$ cross-correlation spectra (Hanning-smoothed over three channels). Labels are the same as in (b). (d) Comet Q4 emission contours from $\mathrm{CH}_{3} \mathrm{OH}$. Contours indicate the $\mathrm{CH}_{3} \mathrm{OH}$ emission near its peak, centered at a cometocentric velocity of 0 km s${ }^{-1}$. The contour levels are $-0.1,0.15,0.2$, and $0.25 \mathrm{Jy}_{\text {beam }}^{-1}(1 \sigma$ spacing, starting at $3 \sigma)$. Image coordinates and labels are the same as in $(a)$. $(e) \mathrm{CH}_{3} \mathrm{OH}$ cross-correlation spectra. Labels are the same as in $(b), \sigma \sim 0.05 \mathrm{Jy}_{\text {beam }}^{-1}$. ( $f$ ) $\mathrm{CH}_{3} \mathrm{OH}$ cross-correlation spectra (Hanning-smoothed over three channels). Abscissa and ordinate are the same as in $(b)$.

molecular species, column (2) lists the transition, column (3) lists the transition frequency (MHz), column (4) lists the upper state energy level of the transition (K), column (5) lists the line strength multiplied by the square of the electronic dipole moment $\left(D^{2}\right)$, and column (6) lists the quiet-Sun photodissociation rate for each species $\left(\mathrm{s}^{-1}\right)$. Table 2 lists the observational parameters. For each comet, the heliocentric distance $\left(r_{H}\right)$, geocentric distance $(\Delta)$ in astronomical units (AU), and the synthesized beam size $\left(\Theta_{a} \times \Theta_{b}\right)$ are listed.

Figures $1 a-1 c$ display the map and spectra of $\mathrm{CH}_{3} \mathrm{OH}$ around comet T7 LINEAR. Figure $1 a$ shows the contour image of the $3_{1,3}-4_{0,4} A^{+}$transition of $\mathrm{CH}_{3} \mathrm{OH}$ starting at $3 \sigma$. Figure $1 b$ shows the cross-correlation spectrum of this transition. The dashed line corresponds to the rest frequency of the $3_{1,3}-4_{0,4} A^{+}$line for a

$\operatorname{CS} 2-1$
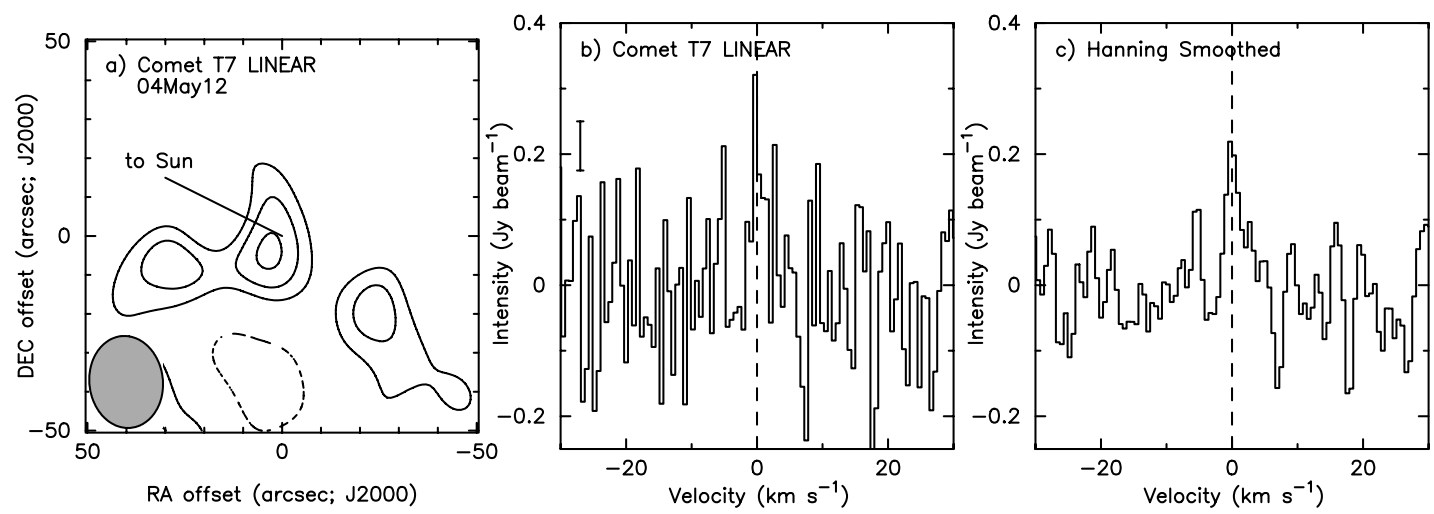

FIG. 2.-Comet LINEAR (C/2002 T7) single-field CS image and spectra. (a) Comet T7 emission contours from the $J=2-1$ transition of CS at $97.980 \mathrm{GHz}$. Contours indicate the CS emission near its peak, centered at a cometocentric velocity of $0 \mathrm{~km} \mathrm{~s}^{-1}$. The contour levels are $-0.15,0.15,0.225$, and $0.3 \mathrm{Jy}$ beam ${ }^{-1}(1 \sigma$ spacing, starting at $2 \sigma$ ). Image coordinates and labels are the same as in Fig. 1. (b) CS cross-correlation spectra. Labels are the same as in Fig. $1 b, \sigma \sim 0.075 \mathrm{Jy}$ beam ${ }^{-1}$. (c) CS cross-correlation spectra (Hanning-smoothed over three channels). Labels are the same as in $(b)$. 
TABLE 3

Variable Temperature and Outflow Velocity (VTOV) Results for Comet Linear

\begin{tabular}{|c|c|c|c|c|c|c|}
\hline $\begin{array}{c}\text { Species } \\
\text { (1) }\end{array}$ & $\begin{array}{c}I_{0} \\
\left(\mathrm{Jy} \mathrm{bm}^{-1}\right) \\
(2)\end{array}$ & $\begin{array}{c}\Delta v \\
\left(\mathrm{~km} \mathrm{~s}^{-1}\right) \\
(3)\end{array}$ & $\begin{array}{c}\left\langle N_{T}\right\rangle \\
\left(\mathrm{cm}^{-2}\right) \\
(4)\end{array}$ & $\begin{array}{c}Q \\
\left(\mathrm{~s}^{-1}\right) \\
(5)\end{array}$ & $\frac{Q(\mathrm{X})}{\underset{(6)}{Q\left(\mathrm{H}_{2} \mathrm{O}\right)}}$ & $\frac{Q(\mathrm{X})}{Q(\mathrm{CN})}$ \\
\hline $\mathrm{CH}_{3} \mathrm{OH} \ldots \ldots \ldots \ldots \ldots \ldots \ldots \ldots \ldots \ldots$ & $0.44(0.06)$ & $1.74(0.31)$ & $1.4(0.3) \times 10^{14}$ & $7.5(1.5) \times 10^{27}$ & $3.8(0.8) \times 10^{-2}$ & \\
\hline CS & $0.21(0.05)$ & $2.45(0.60)$ & $1.4(0.4) \times 10^{12}$ & $1.5(0.5) \times 10^{27}$ & $7.5(2.5) \times 10^{-3}$ & \\
\hline $\mathrm{CH}_{3} \mathrm{CN}$ & $<0.13$ & 1.06 & $<4.6 \times 10^{11}$ & $<2.3 \times 10^{25}$ & $<1.2 \times 10^{-4}$ & $<0.16$ \\
\hline${ }^{13} \mathrm{CO}$ & $<0.12$ & 1.06 & $<1.5 \times 10^{14}$ & $<7.7 \times 10^{27}$ & $<4.0 \times 10^{-2}$ & \\
\hline $\mathrm{CH}_{3} \mathrm{OCHO}$ & $<0.09$ & 1.30 & $<2.5 \times 10^{13}$ & $<2.2 \times 10^{27}$ & $<1.0 \times 10^{-2}$ & \\
\hline HNC & $<0.08$ & 1.35 & $<1.8 \times 10^{11}$ & $<1.4 \times 10^{25}$ & $<7.5 \times 10^{-5}$ & $<0.10$ \\
\hline $\mathrm{HN}^{13} \mathrm{C}$ & $<0.08$ & 1.35 & $<2.8 \times 10^{11}$ & $<2.2 \times 10^{25}$ & $<1.1 \times 10^{-4}$ & $<0.16$ \\
\hline $\mathrm{SiO}$ & $<0.08$ & 1.35 & $<2.2 \times 10^{11}$ & $<1.7 \times 10^{25}$ & $<8.5 \times 10^{-5}$ & \\
\hline $\mathrm{CH}_{3} \mathrm{CH}_{2} \mathrm{CN}$ & $<0.08$ & 1.30 & $<2.3 \times 10^{12}$ & $<1.8 \times 10^{26}$ & $<9.0 \times 10^{-4}$ & $<1.3$ \\
\hline $\mathrm{CH}_{3} \mathrm{CH}_{2} \mathrm{OH}$ & $<0.08$ & 1.35 & $<1.9 \times 10^{13}$ & $<1.4 \times 10^{27}$ & $<7.0 \times 10^{-3}$ & \\
\hline
\end{tabular}

${ }^{\text {a }}$ The ratio is given only for those species that contain a $\mathrm{C}-\mathrm{N}$ bond.

cometocentric rest velocity of $0 \mathrm{~km} \mathrm{~s}^{-1}$. The $1 \sigma \mathrm{rms}$ noise level is indicated at the left of the panel. The $\mathrm{CH}_{3} \mathrm{OH}$ line for comet T7 LINEAR was fit with a Gaussian by a least-squares method, which gives a peak intensity of $0.44(0.06) \mathrm{Jy}^{-1} \mathrm{beam}^{-1}$ and a FWHM of $1.74(0.31) \mathrm{km} \mathrm{s}^{-1}$. Figure $1 c$ shows the crosscorrelation spectrum (Hanning-smoothed over three channels) of this transition. Figures $1 d-1 f$ display the map and spectra of $\mathrm{CH}_{3} \mathrm{OH}$ around comet Q4 NEAT. Figure $1 d$ shows the contour image of $\mathrm{CH}_{3} \mathrm{OH}$ emission starting at $3 \sigma$. Figure $1 e$ is the crosscorrelation spectrum, and the dashed line is similar to Figure $1 b$ for a cometocentric rest velocity of $0 \mathrm{~km} \mathrm{~s}^{-1}$. The least-squares Gaussian line fit for comet Q4 NEAT gives a peak intensity of $0.31(0.13) \mathrm{Jy} \mathrm{beam}^{-1}$ and a FWHM of $0.72(0.3) \mathrm{km} \mathrm{s}^{-1}$. Figure $1 f$ is the Hanning-smoothed cross-correlation spectrum.

Figures $2 a-2 c$ display the map and spectra of CS around comet T7 LINEAR. Figure $2 a$ shows the contour image of the $J=2-1$ transition of CS starting at $2 \sigma$. Figure $2 b$ is the cross-correlation spectrum of this transition. The least-squares Gaussian line fit for comet T7 LINEAR gives a peak intensity of $0.21(0.05) \mathrm{Jy} \mathrm{beam}^{-1}$ and a FWHM of $2.45(0.60) \mathrm{km} \mathrm{s}^{-1}$. Figure $2 c$ is the Hanningsmoothed cross-correlation spectrum.

All other molecular transitions in Table 1, including CS in comet Q4 NEAT, were not detected above $3 \sigma$. Thus, all column density and production rate upper limits were calculated using the $1 \sigma \mathrm{rms}$ noise level of the window containing the molecular line emission and a line width of one channel. Tables 3 and 4 list either the measured intensities (Jy beam ${ }^{-1}$ ) and line widths $\left(\mathrm{km} \mathrm{s}^{-1}\right)$ or the upper limits of the intensity and line width for each species, in columns (2) and (3). Column (4) lists the total beam-averaged molecular column density $\left(\mathrm{cm}^{-2}\right)$ and column (5), the production rate $\left(\mathrm{s}^{-1}\right)$. The calculated production rates were determined using the variable temperature and outflow velocity (VTOV) model (Friedel et al. 2005). Finally, columns (6) and (7) list the production rate ratios with respect to $\mathrm{H}_{2} \mathrm{O}$ and $\mathrm{CN}$, as is discussed in $\S 3.2$.

\section{DISCUSSION}

\subsection{Column Densities and Production Rates}

As described in Friedel et al. (2005), the VTOV model calculates the total column density and production rate of a cometary species, assuming optically thin emission in LTE and that the temperature and outflow velocity within the coma vary with cometocentric distance. The VTOV model calculations to determine the total column density and production rate are discussed in detail in Friedel et al. (2005, Appendix A).

Here we discuss the implication of the production rates and column densities. Using the VTOV model, Tables 3 and 4 list the total beam-averaged column densities and production rates of each molecular species observed toward comets T7 LINEAR and Q4 NEAT. The VTOV model was also used to determine the total beam-averaged column density and production rate of CS, even though it is believed to be the daughter species. The parent molecule of CS may be $\mathrm{CS}_{2}$, which has a very short lifetime of $\sim 10^{3} \mathrm{~S}$ or less (Snyder et al. 2001). This short lifetime suggests that CS is formed in the inner coma and that the measured line width of CS in comet LINEAR is similar to $\mathrm{HCN}$ and $\mathrm{CH}_{3} \mathrm{OH}$, which are known parent species. Thus, at the spatial resolution of our observations, CS can be fitted as though it were a parent species.

\subsection{Relative Production Rates of $\mathrm{X}$ to $\mathrm{H}_{2} \mathrm{O}$ and $\mathrm{CN}$}

\subsubsection{Comet LINEAR}

Based on the $\mathrm{H}_{2} \mathrm{O}$ production rates measured by Schleicher et al. (2005, private communication) at different heliocentric distances, Friedel et al. (2005) determined the $\mathrm{H}_{2} \mathrm{O}$ production

TABLE 4

Variable Temperature and Outflow Velocity (VTOV) Results for Comet NEAT

\begin{tabular}{|c|c|c|c|c|c|c|}
\hline $\begin{array}{l}\text { Species } \\
\text { (1) }\end{array}$ & $\begin{array}{c}I_{0} \\
\left(\mathrm{Jy} \mathrm{bm}^{-1}\right) \\
(2)\end{array}$ & $\begin{array}{c}\Delta v \\
\left(\mathrm{~km} \mathrm{~s}^{-1}\right) \\
(3)\end{array}$ & $\begin{array}{c}\left\langle N_{T}\right\rangle \\
\left(\mathrm{cm}^{-2}\right) \\
(4)\end{array}$ & $\begin{array}{c}Q \\
\left(\mathrm{~s}^{-1}\right) \\
(5)\end{array}$ & $\frac{Q(\mathrm{X})}{Q\left(\mathrm{H}_{2} \mathrm{O}\right)}$ & $\frac{Q(\mathrm{X})}{Q(\mathrm{CN})}{ }^{\mathrm{a}}$ \\
\hline 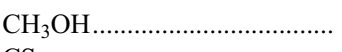 & $0.31(0.13)$ & $0.72(0.30)$ & $4.6(2.7) \times 10^{13}$ & $2.9(1.7) \times 10^{27}$ & $2.4(1.4) \times 10^{-2}$ & \\
\hline CS & $<0.14$ & 0.60 & $<4.0 \times 10^{11}$ & $<1.4 \times 10^{25}$ & $<1.4 \times 10^{-3}$ & \\
\hline $\mathrm{CH}_{3} \mathrm{CN}$ & $<0.06$ & 1.06 & $<2.0 \times 10^{11}$ & $<1.1 \times 10^{25}$ & $<9.2 \times 10^{-5}$ & $<4.0 \times 10^{-2}$ \\
\hline${ }^{13} \mathrm{CO}$ & $<0.06$ & 1.06 & $<9.8 \times 10^{13}$ & $<8.3 \times 10^{27}$ & $<7.0 \times 10^{-2}$ & \\
\hline
\end{tabular}

${ }^{\text {a }}$ The ratio is given only for those species that contain a $\mathrm{C}-\mathrm{N}$ bond. 
rate to be $\sim 2 \times 10^{29} \mathrm{~s}^{-1}$ during our observing period. The relative production rates of each molecular species relative to $\mathrm{H}_{2} \mathrm{O}$ are listed in column (6) of Table 3. The relative production rate ratios of $\mathrm{HCN}$ (Friedel et al. 2005), $\mathrm{CH}_{3} \mathrm{OH}$, and $\mathrm{CS}$ with respect to $\mathrm{H}_{2} \mathrm{O}$ for comet LINEAR are most similar to those observed around comet Hale-Bopp (C/1995 O1) [(2.1-2.6) $\times 10^{-3}, 4 \times$ $10^{-2}$, and $(3.7-4.3) \times 10^{-3}$, respectively; Snyder et al. 2001; Remijan et al. 2004]. Schleicher et al. (2005, private communication) estimated the $\mathrm{CN}$ production rate to be $\sim 1.4 \times 10^{26} \mathrm{~s}^{-1}$. We also attempted to determine the relative production rates of each nitrogen-bearing molecular species relative to $\mathrm{CN}$. However, in this work, no species observed toward comet LINEAR with a CN bond was detected above the $3 \sigma$ detection limit. The upper limits to the production rate ratios are listed in column (7) of Table 3.

\subsubsection{Comet NEAT}

Schleicher et al. (2005, private communication) also determined the $\mathrm{H}_{2} \mathrm{O}$ and $\mathrm{CN}$ production rates for comet NEAT during our observing period (as reported by Friedel et al. 2005). These are very similar to water production rates measured from comet Hyakutake at similar heliocentric distances $\left(\sim 1 \times 10^{29} \mathrm{~s}^{-1}\right.$; Lis et al. 1997). The relative production rates of each molecular species relative to $\mathrm{H}_{2} \mathrm{O}$ are listed in column (6) of Table 4. The relative production rate ratios of $\mathrm{HCN}$ (Friedel et al. 2005) and $\mathrm{CH}_{3} \mathrm{OH}$ relative to $\mathrm{H}_{2} \mathrm{O}$ are most similar to those measured around comet Hyakutake (C/1996 B2;1.2 $\times 10^{-3}$ and $1.7 \times 10^{-2}$, respectively; Biver et al. 1999). The average $\mathrm{CN}$ production rate is $Q(\mathrm{CN}) \sim 2.6 \times 10^{26} \mathrm{~s}^{-1}$. The relative production rates of each nitrogen-bearing molecular species relative to $\mathrm{CN}$ are listed in column (7) of Table 4; however, the only species observed toward comet NEAT with a $\mathrm{CN}$ bond was $\mathrm{CH}_{3} \mathrm{CN}$, which was not detected above the $3 \sigma$ detection limit. The upper limit to the production rate ratio was $<4.0 \times 10^{-2}$.

\subsection{Classifying Comets T7 LINEAR and Q4 NEAT}

Comets are diverse objects, as had been noted by many researchers in the past. For example, A'Hearn et al. (1995) identified the chemical diversity in comets through an optical study of 85 comets. Biver et al. (2002) reached a similar conclusion based on a radio survey of 24 comets, while Mumma et al. (2005) reached the same conclusion from the hypervolatile gases $\mathrm{CO}$ and $\mathrm{CH}_{4}$ from observations at IR wavelengths. Many comet observers have searched for differences between comets from the Oort Cloud and the short-period (Jupiter family) comets to attempt to find clear differences in chemical composition depending on their place of formation. As more observations become available, it becomes harder to differentiate between the various comet classes, in particular after the Deep Impact mission (e.g., Mumma et al. 2005).

We can now include comets T7 LINEAR and Q4 NEAT in the chemical comparison from the detections of both $\mathrm{CH}_{3} \mathrm{OH}$ and HCN (Friedel et al. 2005). Both comets T7 LINEAR and Q4 NEAT are believed to be Oort Cloud comets, given their measured orbital parameters, and our observations show that both comets are chemically very similar. However, from the Biver et al. (2002) $30 \mathrm{~m}$ survey, there appears to be a distinction between comets rich in $\mathrm{HCN}\left(\mathrm{HCN} / \mathrm{H}_{2} \mathrm{O}>0.2 \%\right)$, including comets Hale-
Bopp (C/1995 01), 109P/Swift-Tuttle, 1P/Halley and 9P/Tempel 1 (Mumma et al. 2005), compared to comets with an $\mathrm{HCN} / \mathrm{H}_{2} \mathrm{O}$ abundance ratio $\sim 0.1 \%$, which include, among several others, comets Hyakutake (C/1996 B2), Austin (C/1989 X1), and Levy (C/1990 K1). It is unclear, however, due to such a small sample size, whether in fact there are two distinct classes of comets (i.e., Hale-Bopp and Hyakutake classes) or if comets have a range of different molecular production rate ratios. Given the abundance ratios of comet T7 LINEAR, it is more similar to comet HaleBopp, which was both $\mathrm{HCN}$ - and $\mathrm{CH}_{3} \mathrm{OH}$-rich. Comet Q4 NEAT, on the other hand, has column densities of $\mathrm{HCN}$ and $\mathrm{CH}_{3} \mathrm{OH}$, more similar to comet Hyakutake. Furthermore, this distinction appears across both Oort Cloud and Kuiper-Edgeworth Belt comets, as illustrated by recent Deep Impact observations of comet 9P/ Tempel 1 (Mumma et al. 2005).

Thus, while our observations still tend to support a chemical distinction between Hale-Bopp and Hyakutake class comets due to the abundance rations of $\mathrm{CH}_{3} \mathrm{OH} / \mathrm{H}_{2} \mathrm{O}$ and $\mathrm{HCN} / \mathrm{H}_{2} \mathrm{O}$, more observations of both long- and short-period comets are necessary to increase the sample size. With better statistics, it may be possible to reliably determine the origins of both Oort Cloud and Kuiper-Edgeworth Belt comets in the presolar nebula.

\section{SUMMARY}

In this paper, we have presented the results of an interferometric search for several large molecules, including methanol $\left(\mathrm{CH}_{3} \mathrm{OH}\right)$, methyl cyanide $\left(\mathrm{CH}_{3} \mathrm{CN}\right)$, ethyl cyanide $\left(\mathrm{CH}_{3} \mathrm{CH}_{2} \mathrm{CN}\right)$, ethanol $\left(\mathrm{CH}_{3} \mathrm{CH}_{2} \mathrm{OH}\right)$, and methyl formate $\left(\mathrm{CH}_{3} \mathrm{OCHO}\right)$ in comets $\mathrm{T} 7$ LINEAR and Q4 NEAT. In addition, we also searched for transitions of the simpler molecules $\mathrm{CS}, \mathrm{SiO}, \mathrm{HNC}, \mathrm{HN}^{13} \mathrm{C}$, and ${ }^{13} \mathrm{CO}$. Of the 10 species listed in Table 1, we detected transitions of $\mathrm{CH}_{3} \mathrm{OH}$ and $\mathrm{CS}$ around comet T7 LINEAR and one transition of $\mathrm{CH}_{3} \mathrm{OH}$ around comet Q4 NEAT. Using the VTOV model described by Friedel et al. (2005), we determined the total beamaveraged column densities and production rates of each molecular species observed toward comets T7 LINEAR and Q4 NEAT. Based on the molecular production rate ratios with respect to water, it appears that comet T7 LINEAR is more similar to comet Hale-Bopp, while comet Q4 NEAT is more similar to comet Hyakutake. However, due to such a small sample size, it is unclear whether there are two distinct classes of comet or if there is a continuous range of classes with numerous and different molecular production rate ratios. More observations with higher sensitivity interferometers, such as CARMA or ALMA, of both long- and short-period comets are necessary to increase the sample size, hopefully allowing a determination of the origins of both Oort Cloud and Kuiper-Edgeworth Belt comets in the presolar nebula.

We thank J. R. Dickel for assisting with the observations, an anonymous referee for many helpful comments, and D. G. Schleicher for providing $\mathrm{H}_{2} \mathrm{O}$ and $\mathrm{CN}$ production rates. This work was partially funded by NSF AST 02-28953, AST 0228963, AST 02-28974, and AST 02-28955, and the Universities of Illinois, Maryland, and California, Berkeley, and Caltech.

\section{REFERENCES}

A'Hearn, M. F., Millis, R. L., Schleicher, D. G., Osip, D. J., \& Birch, P. V.

1995, Icarus, 118, 223

Biver, N., et al. 1999, AJ, 118, 1850

2002, Earth Moon Planets, 90, 323

Crovisier, J. 1994, J. Geophys. Res., 99, 3777

Crovisier, J., Bockelée-Morvan, D., Biver, N., Colom, P., Despois, D., \& Lis, D. C. 2004a, A\&A, 418, L35

Crovisier, J., et al. 2004b, A\&A, 418, 1141

Dello Russo, N., Mumma, M. J., DiSanti, M. A., \& Magee-Sauer, K. 2001, BAAS, 33, 1076 
Friedel, D. N., et al. 2005, ApJ, 630, 623

Helbert, J., Rauer, H., Boice, D. C., \& Huebner, W. F. 2005, A\&A, 442, 1107

Hutsemékers, D., Manfroid, J., Jehin, E., Arpigny, C., Cochran, A., Schulz, R., Stüwe, J. A., \& Zucconi, J.-M. 2005, A\&A, 440, L21

Ikeda, M., Kawaguchi, K., Takakuwa, S., Sakamoto, A., Sunada, K., \& Fuse, T. 2002, A\&A, 390, 363

Jewitt, D. C. 2004, in Comets II, ed. M. C. Festou, H. U. Keller, \& H. A Weaver (Tucson: Univ. Arizona Press), 659

Lis, D. C., et al. 1997, Icarus, 130, 355
Mitchell, D. L., et al. 1992, Icarus, 98, 125

Mumma, M. J., et al. 2005, Science, 310, 270

Pickett, H. M., Poynter, R. L., Cohen, E. A., Delitsky, M. L., Pearson, J. C., \&

Müller, H. S. P. 1998, J. Quant. Spectrosc. Radiat. Transfer, 60, 883

Remijan, A., et al. 2004, BAAS, 36, 33.02

Sault, R. J., Teuben, P. J., \& Wright, M. C. H. 1995, in ASP Conf. Ser. 77, Astronomical Data Analysis Software and Systems IV (San Francisco: ASP), 433 Snyder, L. E., et al. 2001, AJ, 121, 1147

Weissman, P. R. 1999, Space Sci. Rev., 90, 301 